\title{
Emergency department antibiotic use for exacerbations of COPD
}

\section{Aleksandr M Tichter' Grigory Ostrovskiy ${ }^{2}$}

'New York Presbyterian Hospital, Columbia University Medical Center, VC-2, New York, NY, USA; ${ }^{2}$ Department of Medical Education, Weill Cornell Medicine-Qatar, Qatar Foundation - Education City, Doha, Qatar
Correspondence: Aleksandr M Tichter Department of Emergency Medicine, New York Presbyterian Hospital, Columbia University Medical Center, 622 West 168th Street, VC-2, New York, NY 10032, USA

Tel +l 2123052995

Email amt2116@cumc.columbia.edu
This article was published in the following Dove Press journal: Open Access Emergency Medicine

Background: COPD is the third leading cause of death, with acute exacerbations accounting for 1.5 million emergency department (ED) visits annually. Guidelines include recommendations for antibiotic therapy, though evidence for benefit is limited, and little is known about ED prescribing patterns. Our objectives were to determine the rate with which ED patients with acute exacerbations of COPD (AECOPD) are treated with antibiotics, compare the proportions of antibiotic classes prescribed, describe trends of antibiotic treatment, and identify predictors of antibiotic therapy.

Patients and methods: This was an analysis of the National Hospital Ambulatory Medical Care Survey (NHAMCS) for the years 2009-2014. Descriptive statistics were used to summarize the rate of antibiotic therapy and the relative proportions of each antibiotic class prescribed for AECOPD. Logistic regression was used to measure the trend in treatment rate over time and identify the variables associated with antibiotic use.

Results: There were an estimated 4.5 million ED visits for AECOPD. Antibiotic treatment occurred at a rate of $39 \%$. Among those treated, macrolides (41\%) and quinolones (35\%) were prescribed most frequently. Logistic regression did not reveal a trend in antibiotic treatment over time and identified emergent/immediate triage level (OR 2.11,95\% CI 1.09-4.10) and elevated temperature (OR 7.92, 95\% CI 2.28-27.50) as being independently associated with antibiotic therapy.

Conclusion: Less than half of the ED visits for AECOPD resulted in antibiotic therapy, with no upward trend over time. Fever and triage level were predictive of antibiotic therapy, with macrolides and quinolones constituting the agents most commonly prescribed.

Keywords: humans, chronic obstructive pulmonary disease, exacerbation, anti-bacterial agents, cross-sectional studies, trend

\section{Introduction}

COPD is characterized by a combination of small airway inflammation and loss of lung parenchymal elasticity which results in air trapping and progressive airflow limitation. ${ }^{1}$ It is the third leading cause of death and has up to a $10 \%$ prevalence worldwide. ${ }^{2}$ Due to ongoing exposure to risk factors, which include cigarette smoking, air pollution, and age, the global burden of COPD is expected to increase. ${ }^{1}$ Acute exacerbations of COPD account for 1.5 million emergency department (ED) visits and \$11.3 billion in hospital costs annually. They have an adverse effect on both quality of life and prognosis, with an estimated $49 \%$ all-cause mortality within 3 years of hospitalization.,

Therapeutic mainstays include inhaled short-acting bronchodilators and systemic corticosteroids. Guidelines for the ED management of COPD exacerbations 
additionally recommend expanding treatment to include antibiotics, administration of which has been previously identified as a quality indicator. ${ }^{5}$ The rationale for empiric antibiotic therapy is based on an assumption of bacterial causality, together with a desire to avoid infectious complications. However, the utility of antibiotics in the ED population remains unclear. The trials which have demonstrated a reduction in treatment failure showed small and inconsistent effects and did not enroll patients from the ED. Evidence for mortality benefit is restricted to intensive care unit (ICU) patients with limited generalizability. ${ }^{6}$

Antibiotic use for acute exacerbations of COPD (AECOPD) remains controversial, and little is known regarding ED prescribing patterns. The aims of this study, therefore, were to determine the rate at which ED patients with AECOPD are treated with antibiotics, compare the relative proportions of antibiotic classes prescribed, describe trends of antibiotic treatment over the study period, and identify independent predictors of antibiotic therapy.

\section{Patients and methods}

\section{Study design}

This study is a secondary analysis of the National Hospital Ambulatory Medical Care Survey (NHAMCS). Conducted by the Centers for Disease Control and Prevention, National Center for Health Statistics (NCHS), the NHAMCS is an annual sample of ambulatory visits made to non-federal, general, and short-stay hospitals in the US. The scope, sample design, data collection and processing, and estimation procedures for the NHAMCS are available from the NCHS. ${ }^{7}$ Briefly, NHAMCS uses a four-stage probability design to randomly sample geographic regions as primary sampling units, hospitals within primary sampling units, EDs within hospitals, and visits within EDs. There is one probability for each sampling stage, with the overall probability of selection being the product of the probability at each stage. Once a sampled hospital consents to participation in the NHAMCS, a US Census Bureau field representative is sent to train staff on the data collection process. This process was conducted manually until 2012, since which time the NHAMCS has implemented an automated, computerized survey tool. The target number of sampled records is 100 per ED over the course of a randomly assigned 4-week reporting period, with each being assigned a weight that is equal to the inverse of its probability of being included in the sample, and which indicates how many ED visits in the entire US that visit represents. ${ }^{8-10}$ Because this study involves existing, de-identified, and publicly available data, it was determined by the institutional review board at
Columbia University Medical Center to qualify for exempt status (IRB-AAAQ2211).

\section{Study setting and population}

Although the NHAMCS includes visits to selected ambulatory care departments, this analysis focuses solely on the visits to hospital EDs between the years 2009 and 2014. The population was defined as visits by patients over the age of 24 for AECOPD, identified by ED primary diagnoses corresponding to ICD-9 codes 491.21 (obstructive chronic bronchitis with [acute] exacerbation), 491.22 (obstructive chronic bronchitis with acute bronchitis), 491.9 (unspecified chronic bronchitis), 492.8 (other emphysema), and 496.0 (chronic airway obstruction, not elsewhere classified). Visits were excluded if any secondary diagnoses corresponded to ICD-9 codes specifying bacterial pulmonary infections: 481.0 (pneumococcal pneumonia), 482.1 (pneumonia due to pseudomonas), 482.42 (methicillin-resistant Staphylococcus aureus pneumonia), 482.9 (unspecified bacterial pneumonia), 485.0 (unspecified bronchopneumonia), and 486.0 (unspecified pneumonia).

\section{Measurements and variables}

The primary outcome was antibiotic treatment, either in the ED or prescribed upon discharge, using Multum Lexicon level 2 therapeutic drug categories 009 (cephalosporins), 011 (macrolide derivatives), 012 (miscellaneous antibiotics), 013 (penicillins), 014 (quinolones), 015 (sulfonamides), 016 (tetracyclines), 018 (aminoglycosides), and 406 (glycopeptide antibiotics).

The main predictor was year of ED visit, with age, gender, race/ethnicity (white only, non-Hispanic; black only, non-Hispanic; Hispanic; or other, non-Hispanic), expected source of payment (private insurance or non-private insurance), US region (Northeast, Midwest, South, or West), means of arrival (ambulance or non-ambulance), triage level (immediate, emergent, urgent, semi-urgent, or non-urgent), temperature $\left(\leq 94.9^{\circ} \mathrm{F}, 95-100.3^{\circ} \mathrm{F}, \geq 100.4^{\circ} \mathrm{F}\right)$, and $\mathrm{ED}$ disposition (discharged, admitted to floor, admitted to the ICU, or deceased in ED) comprising the covariates. Missing values for age, gender, and race/ethnicity were imputed by the NCHS. For triage level, responses were rescaled by the NCHS to fit a five-level triage system. The rescaling method was determined in consultation with subject matter experts and based on record analysis. Missing responses for triage level were imputed from 2009 to 2011 , but not from 2012 to 2014. ${ }^{11}$ Records with missing values for the expected source of payment, US region, means of arrival, temperature, triage level, and disposition were excluded from the analysis. 


\section{Statistical analyses}

All analyses were performed using Stata 14.2 (StataCorp, College Station, TX, USA) and adhered to recommended NHAMCS procedures. ${ }^{12}$ Sampled visit weights were analyzed, which have been adjusted by NCHS for survey nonresponse, yielding an unbiased national estimate of ED visit occurrences, percentages, and characteristics. Estimates based on $<30$ observations or with $>30 \%$ relative standard error were considered unreliable and labeled as such in the relevant data tables and figures. The variables to which this applied included race/ethnicity (other, non-Hispanic), triage level (immediate and non-urgent), and disposition (deceased). These variables were additionally expressed in a re-coded format, which collapsed those subcategories with either $<30$ observations or $>30 \%$ relative standard error in order to ensure estimate reliability.

Descriptive statistics were used to summarize both average overall and yearly proportions of any antibiotic therapy, as well as the relative proportions of each antibiotic class prescribed, by percentages of total with two-sided CIs. For the aims of determining the significance of trend over time and identifying predictors of antibiotic therapy, the primary outcome variable was dichotomized, and a multivariable logistic model was constructed to regress antibiotic therapy on year of ED visit, which was treated as continuous. We decided a priori to include all the covariates in the multivariable model; no stepwise selection procedures were considered.

\section{Results}

There were 999 total records in NHAMCS which met the inclusion criteria, corresponding to 4.5 million presentations for AECOPD and representing 0.56\% (95\% CI $0.50 \%-0.63 \%$ ) of all ED visits from 2009 to 2014 . The primary diagnoses which accounted for the largest proportion included chronic bronchitis with acute exacerbation (57\%) and COPD (35\%).

The majority of visits occurred in the South $(39.79 \%)$, among patients who were female (58.33\%), in the 45-64 age group (43.40\%), non-privately insured (66.08\%), and who did not arrive by ambulance (61.24\%). A majority of the visits were triaged as "urgent" $(52.05 \%)$ and ultimately resulted in discharge (63.09\%). The demographic characteristics of the population together with the estimates of the numbers of weighted visits are shown in Table 1.

Antibiotic treatment occurred at an estimated rate of $39.07 \%$ (95\% CI 34.34-44.01) overall. Among cases in which an antibiotic was given, macrolides $(40.67 \%, 95 \%$ CI
33.05-48.76) and quinolones (34.89\%, 95\% CI 28.50-41.86) were used most frequently (Figure 1).

\section{Trend over time}

Between 2009 and 2014, there was a non-significant trend toward a decreased proportion of ED visits for AECOPD ( $18.52 \%$ vs $17.83 \%$, unadjusted $P=0.88$ ), with a corresponding non-significant trend toward increased rates of antibiotic treatment overall $(38.67 \%$ vs $43.12 \%$, unadjusted $P$-value 0.53 ; Figure 2). In an unadjusted analysis of antibiotic treatment trend across disposition subgroups, only those records which resulted in ICU admission or death showed a significant association with the year of visit, with a reduction in the odds of antibiotic therapy between 2009 and 2014 (OR $0.53,95 \%$ CI 0.31-0.92).

After adjusting for age, gender, race/ethnicity, insurance status, region, means of arrival, triage level, temperature, and disposition, however, there remained no significant association between the year of ED visit and antibiotic treatment (OR 0.98, 95\% CI 0.87-1.11).

\section{Characteristics associated with antibiotic treatment}

In the multivariable analysis, emergent/immediate triage level (OR 2.11, 95\% CI 1.09-4.10) and elevated temperature (OR 7.92, 95\% CI 2.28-27.50) were independently associated with increased odds of antibiotic treatment. None of the variables included in the model were independently associated with reduced odds of antibiotic treatment (Table 2).

\section{Discussion}

The benefit of antibiotics for AECOPD is unclear, and there have been no recent studies examining prescribing practices specific to the ED population. Using data from a national sample of ED visits, we found an overall treatment rate of $39 \%$, with no significant change in antibiotic use across our 2009-2014 study period.

Fever was the strongest independent predictor for antibiotic therapy in our multivariable model, with the odds of antibiotic prescription nearly eight times higher among patients with a temperature greater than or equal to 100.4 compared with those who were normothermic. These results suggest that, rather than using empiric antibiotics for the avoidance of infectious complications in AECOPD, providers reserve treatment for cases in which objective signs of infection already exist. One possible explanation for this practice is the heightened vigilance with respect to and an inclination 
Table I Demographic characteristics of patients who visited the ED for AECOPD

\begin{tabular}{|c|c|c|c|c|}
\hline Variables & $\begin{array}{l}\text { Number of } \\
\text { unweighted visits }\end{array}$ & $\begin{array}{l}\text { Number of } \\
\text { weighted visits }\end{array}$ & $\begin{array}{l}\text { Weighted proportion } \\
\text { of visits, \% } \\
(95 \% \mathrm{Cl})\end{array}$ & $\begin{array}{l}\text { Weighted proportion } \\
\text { receiving antibiotic } \\
\text { treatment, \% } \\
(95 \% \mathrm{Cl})\end{array}$ \\
\hline Total visits & 999 & $4,500,000$ & $0.56(0.50-0.63)$ & $39.07(34.34-44.01)$ \\
\hline \multicolumn{5}{|l|}{ Year } \\
\hline 2009 & 205 & 840,000 & $18.52(13.73-24.52)$ & $38.67(27.86-50.72)$ \\
\hline 2010 & 184 & 710,000 & I5.7I (II.96-20.36) & $46.35(39.09-53.77)$ \\
\hline 2011 & 179 & 740,000 & $16.49(12.8 \mathrm{I}-20.98)$ & $43.89(33.5 \mathrm{I}-54.83)$ \\
\hline 2012 & 168 & 630,000 & $13.95(10.47-18.35)$ & $29.87(21.14-40.35)$ \\
\hline 2013 & $|4|$ & 790,000 & $17.5(13.06-23.04)$ & $31.63(22.6 I-42.30)$ \\
\hline 2014 & 122 & 800,000 & $17.83(13.09-23.80)$ & $43.12(32.13-54.82)$ \\
\hline \multicolumn{5}{|l|}{ Age category, years } \\
\hline $25-44$ & 42 & 180,000 & $4.08(2.79-5.93)$ & $45.5(27.44-64.83)$ \\
\hline $45-64$ & 416 & $2,000,000$ & $43.4(39.10-47.80)$ & $41.66(34.89-48.76)$ \\
\hline $65-74$ & 286 & $1,300,000$ & $28.72(25.03-32.73)$ & $38.36(29.6 I-47.93)$ \\
\hline 75 and older & 252 & $\mathrm{I}, 100,000$ & $23.8(20.14-27.88)$ & $34.36(26.19-43.57)$ \\
\hline \multicolumn{5}{|l|}{ Gender } \\
\hline Male & 444 & $1,900,000$ & $41.67(37.89-45.56)$ & $40.22(33.15-47.72)$ \\
\hline Female & 555 & $2,600,000$ & $58.33(54.44-62.11)$ & $38.25(32.79-44.03)$ \\
\hline \multicolumn{5}{|c|}{ Race/ethnicity (collapsed) } \\
\hline White & 787 & $3,600,000$ & $80(75.43-83.89)$ & $40.34(34.77-46.16)$ \\
\hline Black & 139 & 580,000 & $12.95(10.08-16.48)$ & $37.93(29.06-47.68)$ \\
\hline Hispanic or others & 73 & 180,000 & $7.06(4.6-10.67)$ & $26.83(16.1 I-4 I .19)$ \\
\hline \multicolumn{5}{|l|}{ Payment } \\
\hline Private & 339 & $\mathrm{I}, 500,000$ & $33.92(29.01-39.19)$ & $39.07(31.60-47.09)$ \\
\hline Non-private & 660 & $3,000,000$ & $66.08(60.8 I-70.99)$ & $39.07(32.95-45.55)$ \\
\hline \multicolumn{5}{|l|}{ US region } \\
\hline Northeast & 201 & 640,000 & $14.2(|\mathrm{I} .27-| 7.75)$ & $38.4 I(27.6 I-50.48)$ \\
\hline Midwest & 296 & 130,000 & $28.45(23.29-34.24)$ & $42.19(35.27-49.43)$ \\
\hline South & 341 & $1,800,000$ & $39.79(33.39-46.56)$ & $38.46(29.80-47.92)$ \\
\hline West & 161 & 790,000 & $17.56(13.77-22.12)$ & $35.93(26.99-45.97)$ \\
\hline \multicolumn{5}{|l|}{ Mode of arrival } \\
\hline EMS & 370 & $1,700,000$ & $38.76(34.18-43.55)$ & $36.58(29.66-44.10)$ \\
\hline Ambulatory & 598 & $2,700,000$ & $61.24(56.45-65.82)$ & $40.17(34.27-46.37)$ \\
\hline \multicolumn{5}{|l|}{ ESI level (collapsed) } \\
\hline Immediate/emergent & 245 & $1,100,000$ & $28.4(24.35-32.82)$ & $44.87(36.14-53.93)$ \\
\hline Urgent & 476 & $2,100,000$ & $52.04(47.74-56.30)$ & $41.19(33.94-48.84)$ \\
\hline Semi-/non-urgent & 146 & 780,000 & $19.57(15.61-24.24)$ & $33.11(23.15-44.85)$ \\
\hline \multicolumn{5}{|c|}{ Temperature category } \\
\hline Hypothermic & 57 & 260,000 & $5.7(4.11-7.87)$ & $41.66(26.47-58.62)$ \\
\hline Normothermic & 905 & $4,100,000$ & $90.8(88.33-92.78)$ & $37.28(32.6 I-42.20)$ \\
\hline Hyperthermic & 37 & 160,000 & $3.5(2.4 I-5.05)$ & $81.29(58.83-92.96)$ \\
\hline \multicolumn{5}{|c|}{ Disposition (collapsed) } \\
\hline Home & 580 & $2,700,000$ & $63.09(57.35-68.48)$ & $38.36(32.33-44.77)$ \\
\hline Floor & 312 & $1,400,000$ & $32.38(27.6 I-37.54)$ & $36.72(29.7 I-44.33)$ \\
\hline ICU/deceased & 49 & 190,000 & $4.54(3.13-6.53)$ & $41.64(24.76-60.74)$ \\
\hline
\end{tabular}

Notes: Unweighted visits include sampled ED records over a prespecified reporting period. Each unweighted visit represents a larger number of ED visits across the US (weighted visits), determined by the inverse of its probability of being sampled.

Abbreviations: AECOPD, acute exacerbation of COPD; ED, emergency department; ESI, emergency services index; ICU, intensive care unit; EMS, emergency medical services.

to align practice with the high-profile campaign for antibiotic stewardship, which began to appear in the emergency medicine literature in a more conspicuous manner at the beginning of our study period. ${ }^{13,14}$ This may similarly account for the temporal plateau in antibiotic use we observed as compared with the steady increase in antibiotic prescription for AECOPD across a preceding study period which followed the publication of COPD guidelines advocating for antibiotics as a complement to the traditional therapeutic mainstays. ${ }^{15}$

Interestingly, our results demonstrate no difference in antibiotic use as a function of disposition destination, with patients admitted to the ICU treated at the same rate as 


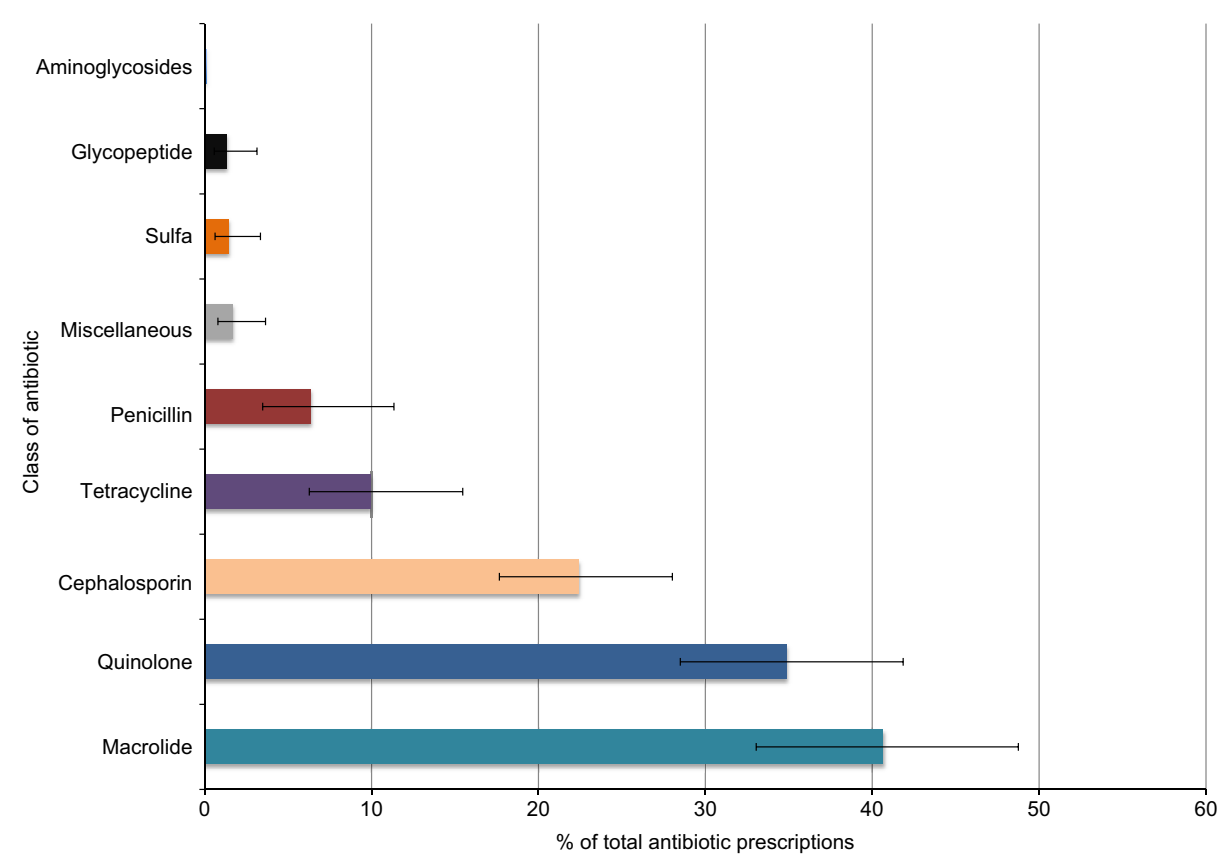

Figure I Relative proportions of antibiotics prescribed.

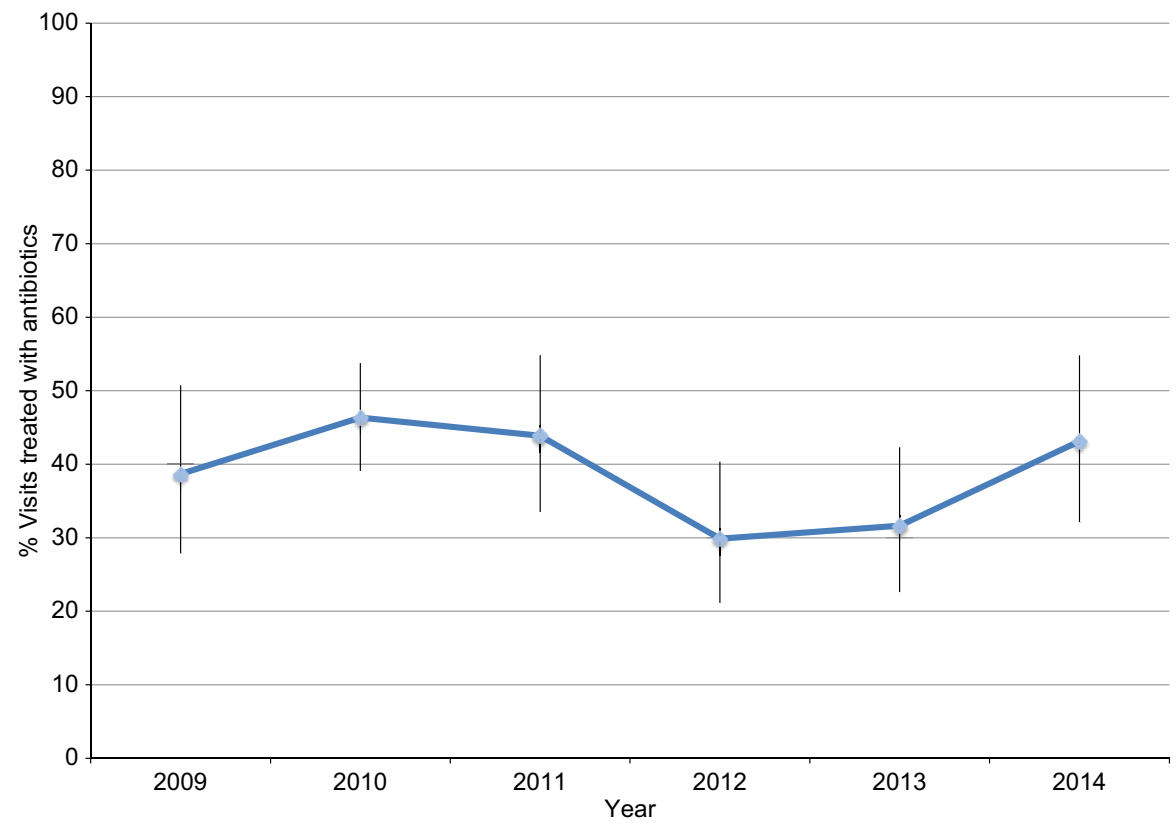

Figure 2 Yearly trend in antibiotic treatment.

those who are discharged home, and disposition destination failing to reach significance as an independent predictor in our regression analysis. This is notable considering the fact that the only group of patients for whom mortality benefit has been established are those with AECOPD admitted to the ICU. ${ }^{16}$ In isolation, this finding suggests that providers do not consider acuity in determining which patients to treat. However, this implication is contradicted by the fact that visits with an emergent/immediate triage level had over double the odds of being treated, compared with non-urgent/ semi-urgent visits. There are several potential explanations for this divergence. First, it is possible that triage level was a poor indicator of critical illness and did not accurately predict which patients ultimately required ICU admission. 
Table 2 Factors associated with antibiotic treatment in multivariable model

\begin{tabular}{|c|c|c|c|}
\hline \multirow{3}{*}{$\begin{array}{l}\text { Variables } \\
\text { Year }\end{array}$} & \multicolumn{3}{|c|}{ Antibiotic treatment } \\
\hline & \multirow{2}{*}{\begin{tabular}{|c|} 
OR \\
0.98
\end{tabular}} & \multicolumn{2}{|c|}{$95 \% \mathrm{Cl}$} \\
\hline & & 0.87 & 1.11 \\
\hline \multicolumn{4}{|l|}{ Age category, years } \\
\hline $25-44$ & Ref. & \multicolumn{2}{|l|}{-} \\
\hline $45-64$ & 0.69 & 0.28 & 1.74 \\
\hline $65-74$ & 0.67 & 0.25 & 1.82 \\
\hline$\geq 75$ & 0.44 & 0.17 & 1.15 \\
\hline \multicolumn{4}{|l|}{ Gender } \\
\hline Female & Ref. & \multicolumn{2}{|l|}{-} \\
\hline Male & 1.01 & 0.66 & 1.56 \\
\hline \multicolumn{4}{|c|}{ Race/ethnicity (collapsed) } \\
\hline White, non-Hispanic & Ref. & \multicolumn{2}{|l|}{-} \\
\hline Black, non-Hispanic & 0.92 & 0.53 & 1.60 \\
\hline Hispanic/others & 0.72 & 0.37 & 1.38 \\
\hline \multicolumn{4}{|l|}{ Payment } \\
\hline Non-private insurance & Ref. & \multicolumn{2}{|l|}{-} \\
\hline Private insurance & 1.11 & 0.68 & 1.82 \\
\hline \multicolumn{4}{|l|}{ US region } \\
\hline Northeast & Ref. & \multicolumn{2}{|l|}{-} \\
\hline Midwest & 1.10 & 0.57 & 2.14 \\
\hline South & 0.85 & 0.43 & 1.68 \\
\hline West & 0.79 & 0.40 & 1.55 \\
\hline \multicolumn{4}{|l|}{ Mode of arrival } \\
\hline Non-EMS & Ref. & \multicolumn{2}{|l|}{-} \\
\hline EMS & 0.89 & 0.58 & 1.39 \\
\hline \multicolumn{4}{|l|}{ ESI level (collapsed) } \\
\hline Semi-/non-urgent & Ref. & \multicolumn{2}{|l|}{-} \\
\hline Urgent & 1.55 & 0.88 & 2.74 \\
\hline Immediate/emergent* & 2.11 & 1.09 & 4.10 \\
\hline \multicolumn{4}{|c|}{ Temperature category } \\
\hline Normothermic & Ref. & \multicolumn{2}{|l|}{-} \\
\hline Hypothermic & 1.57 & 0.70 & 3.53 \\
\hline Hyperthermic* & 7.92 & 2.28 & 27.50 \\
\hline \multicolumn{4}{|c|}{ Disposition (collapsed) } \\
\hline Discharged & Ref. & \multicolumn{2}{|l|}{-} \\
\hline Floor & 0.71 & 0.46 & 1.11 \\
\hline ICU/deceased & 1.07 & 0.46 & 2.49 \\
\hline
\end{tabular}

Note: $* P<0.05$.

Abbreviations: ESI, emergency severity index; ICU, intensive care unit; Ref., reference; EMS, emergency medical services.

This, however, is unlikely given the fact that triage level has previously been proven a reliable and valid tool for assessing resource needs and acuity. ${ }^{17}$ Second, it is possible that patients who are identified at triage as being in need of emergent/immediate attention are aggressively treated early in their clinical course, and their level of care is ultimately de-escalated such that they become candidates for general floor or step-down admission. Finally, it is possible that ED providers make their treatment decisions at the outset of the patient encounter when information regarding triage level is readily available, but fail to escalate therapy if the clinical course deteriorates.
While the evidence for antibiotics for AECOPD comes with caveats, national guidelines should theoretically influence medication choice for those who are ultimately treated. In fact, our data identify macrolides as being prescribed with the highest relative proportion (41\%), which is consistent with the current recommendations. However, whereas aminopenicillins with clavulanic acid and tetracyclines represent the suggested alternatives, we found them to be sparingly utilized in our national sample. Instead, quinolones are the predominant second choice among ED providers (35\%). Although this practice pattern better matches the antibiotic guidelines for the treatment of community-acquired pneumonia, we explicitly excluded patients with a secondary diagnosis of pneumonia from our study population. ${ }^{17}$ This prescribing pattern has two possible explanations. The first is a partial departure from guideline-recommended antibiotic choices when the decision to initiate antibiotic therapy for uncomplicated AECOPD is made. The second is treatment which is reserved only for suspected, but unconfirmed or undocumented bronchopneumonia - a more likely explanation when contextualized by our finding that fever is the strongest independent predictor of antibiotic therapy.

\section{Limitations}

Our study is vulnerable to the constraints of any observational design using large, publicly available data, such as the NHAMCS, including the inability to draw conclusions about causal relationships, and errors during data abstraction. The latter concern is somewhat mitigated by the mechanisms for reliability assurance employed by the NHAMCS..$^{18}$

Accurate identification of the population of interest is limited by several factors. As with any research which relies on pre-existing data, the potential for diagnostic miscoding on the part of providers is a concern. For example, in the event that a definitive diagnosis is not established at the time of the ED encounter, a provider may enter a symptom (eg, shortness of breath), rather than a disease (eg, obstructive chronic bronchitis with acute exacerbation), as the primary ICD-9 code. Additionally, while we attempted to more accurately identify visits for AECOPD by limiting the included ICD-9 codes to the primary ED diagnosis field, this approach would miss relevant codes listed as secondary or tertiary diagnostic entries. It would also be desirable to confirm COPD diagnoses with lung function data, but this variable is not available in the NHAMCS. Our data are substantiated, however, by the fact that the number of ED visits for AECOPD across our study period approximates estimates which have been previously published. ${ }^{15}$ 
Although we report an antibiotic prescription rate of $39.07 \%$, this number may be inaccurate in that it fails to capture patients who were already taking antibiotics at the time of their ED visit and, therefore, would not have had an indication of the fact that they were being treated with antimicrobial agents in any of the medical record fields which are routinely abstracted by the NHAMCS data collectors. Another limitation involves our assumption that any antibiotic prescribed was for the treatment of AECOPD. There is no reliable way to exclude all other indications for antibiotic therapy from our analysis, and although we attempted to mitigate this constraint by specifically excluding visits with ICD-9 codes corresponding to bacterial pulmonary infections, patients may have received antibiotics for the treatment of coincident infections of other organ systems diagnosed at the time of their ED visit.

Finally, one of our objectives was to compare national practice patterns with COPD guidelines. While they do not distinguish between levels of disease severity the way supporting evidence does, the guidelines recommend antibiotic treatment for exacerbations characterized by a specific combination of cardinal COPD symptoms including increased sputum purulence together with either increased dyspnea or sputum volume, none of which are variables available for analysis within the NHAMCS. ${ }^{5}$ We were similarly unable to adjust for comorbidities, and environmental and social factors such as smoking, which may confound the relationship between some of the other covariates and our outcome of interest.

\section{Conclusion}

Our results provide the most recent insight into the epidemiology and treatment of AECOPD in the ED. Less than half of the ED visits result in initiation of antimicrobial therapy, with elevated temperature and emergent/immediate ESI level being the only independent predictors of antibiotic treatment. Among those who are treated, macrolides and quinolones are most commonly prescribed. Although the relative benefit of antibiotics must be weighed against the pitfalls of overuse, they remain a target for quality improvement efforts, particularly for patients admitted to the ICU, for whom mortality benefit is more clear.

\section{Disclosure}

The authors report no conflicts of interest in this work.

\section{References}

1. Hess MW. The 2017 global initiative for chronic obstructive lung disease report and practice implications for the respiratory therapist. Respir Care. 2017;62(11):1492-1500.

2. Halbert RJ, Isonaka S, George D, Iqbal A. Interpreting COPD prevalence estimates: what is the true burden of disease? Chest. 2003;123(5): 1684-1692.

3. Decramer M, Janssens W, Miravitlles M. Chronic obstructive pulmonary disease. Lancet. 2012;379(9823):1341-1351.

4. Hoyert DL. 75 years of mortality in the United States, 1935-2010. NCHS Data Brief. 2012;88(88):1-8.

5. Gold PM. The 2007 GOLD Guidelines: a comprehensive care framework. Respir Care. 2009;54(8):1040-1049.

6. Tichter AM. Are routine antibiotics beneficial for exacerbations of chronic obstructive pulmonary disease? Ann Emerg Med. 2013;62(6):592-593.

7. Centers for Disease Control and Prevention, National Center for Health Statistics [webpage on the Internet]. Ambulatory health care data: questionnaires, datasets, and related documentation. Available from: https:www.cdc.gov/nchs/ahcd/ahcd_questionnaires.htm. Accessed September 21, 2017.

8. Cooper RJ, Schriger DL. Annals of Emergency Medicine Journal Club. The NHAMCS database, chart review methods, and more on regression modeling answers to the May 2010 journal club questions. Ann Emerg Med. 2010;56(4):431-437.

9. Kea B, Fu R, Lowe RA, Sun BC. Interpreting the national hospital ambulatory medical care survey: United States emergency department opioid prescribing, 2006-2010. Acad Emerg Med. 2016;23(2):159-165.

10. Taur Y, Smith MA. Adherence to the Infectious Diseases Society of America guidelines in the treatment of uncomplicated urinary tract infection. Clin Infect Dis. 2007;44(6):769-774.

11. Centers for Disease Control and Prevention, National Center for Health Statistics [webpage on the Internet]. Ambulatory health care data: data collection and processing. Available from: https://www.cdc.gov/nchs/ ahcd/ahcd_data_collection.htm. Accessed September 21, 2017.

12. McCaig LF, Burt CW. Understanding and interpreting the National Hospital Ambulatory Medical Care Survey: key questions and answers. Ann Emerg Med. 2012;60(6):716-721.e1.

13. Pope SD, Dellit TH, Owens RC, Hooton TM; Infectious Diseases Society of America; Society for Healthcare Epidemiology of America. Results of survey on implementation of Infectious Diseases Society of America and Society for Healthcare Epidemiology of America guidelines for developing an institutional program to enhance antimicrobial stewardship. Infect Control Hosp Epidemiol. 2009;30(1):97-98.

14. May L, Cosgrove S, L'Archeveque M, et al. A call to action for antimicrobial stewardship in the emergency department: approaches and strategies. Ann Emerg Med. 2013;62(1):69-77.e2.

15. Tsai CL, Sobrino JA, Camargo CA Jr. National study of emergency department visits for acute exacerbation of chronic obstructive pulmonary disease, 1993-2005. Acad Emerg Med. 2008;15(12):1275-1283.

16. Vollenweider DJ, Jarrett H, Steurer-Stey CA, Garcia-Aymerich J, Puhan MA. Antibiotics for exacerbations of chronic obstructive pulmonary disease. Cochrane Database Syst Rev. 2012;12:CD010257.

17. Mandell LA, Wunderink RG, Anzueto A, et al; Infectious Diseases Society of America; American Thoracic Society. Infectious Diseases Society of America/American Thoracic Society consensus guidelines on the management of community-acquired pneumonia in adults. Clin Infect Dis. 2007;44(Suppl 2):S27-S72.

18. Mccaig L, Mclemore T. Plan and operation of the National Hospital Ambulatory Care Survey National Center for Healthcare Statistics. Vital Health Stat.1994;1(34):6-8. 


\section{Publish your work in this journal}

The Open Access Emergency Medicine is an international, peerreviewed, open access journal publishing original research, reports, editorials, reviews and commentaries on all aspects of emergency medicine. The manuscript management system is completely online and includes a very quick and fair peer-review system, which is all

Submit your manuscript here: https://www.dovepress.com/open-access-emergency-medicine-journal

easy to use. Visit http://www.dovepress.com/testimonials.php to read real quotes from published authors. 